\title{
TRIPLE POSITIVE SOLUTIONS FOR THE ONE-DIMENSIONAL $p$-LAPLACIAN
}

\author{
ZHANBING BAI, MINGFU MA AND XIANGQIAN LIANG
}

\begin{abstract}
We consider the boundary value problem: $\left(\varphi_{p}\left(x^{\prime}(t)\right)\right)^{\prime}+q(t) f\left(t, x(t), x^{\prime}(t)\right)=0, p>$ $1, t \in[0,1]$, with $x(0)=x(1)=0$, or $x(0)=x^{\prime}(1)=0$. Using a fixed point theorem due to Avery and Peterson, sufficient conditions are obtained that guarantee the existence of at least three positive solutions. The emphasis here is the nonlinear term $f$ is involved with the first order derivative. An example is also included to illustrate the importance of the results obtained.
\end{abstract}

\section{Introduction}

Recently, the existence and multiplicity of positive solutions for nonlinear ordinary differential equations as well as difference equations have been studied extensively; to identify a few, we refer the reader to [1-20]. Among them, an interest in triple solutions evolved from the Leggett-Williams multiple fixed-point theorem [13]. And lately, two triple fixed-point theorems due to Avery [2] and Avery and Peterson [6] have been applied to obtain triple solutions of certain boundary value problems for ordinary differential equations as well as for their discrete analogues.

In [6], Avery and Peterson generalize the fixed-point theorem of Leggett-Williams by using theory of fixed-point index and Dugundji extension theorem. An application of the theorem be given to prove the existence of three positive solutions to the following second-order discrete boundary value problem

$$
\begin{gathered}
\Delta^{2} x(k-1)+f(x(k))=0, \quad \text { for all } k \in[a+1, b+1], \\
x(a)=x(b+2)=0,
\end{gathered}
$$

where $f: R \rightarrow[0, \infty)$ is continuous.

In this paper, we shall consider the existence of multiple solutions for the onedimensional singular $p$-Laplace equations:

$$
\left(\varphi_{p}\left(x^{\prime}(t)\right)\right)^{\prime}+q(t) f\left(t, x(t), x^{\prime}(t)\right)=0,0<t<1,
$$

Received and revised July 27, 2004.

2000 Mathematics Subject Classification. 34B15.

Key words and phrases. Triple positive solutions, $p$-Laplacian, boundary value problem, fixedpoint theorem.

This work is sponsored by the National Nature Science Foundation of China (10371006) and the Doctoral Program Foundation of Education Ministry of China (1999000722). 
subject to one of the following boundary conditions:

$$
x(0)=x(1)=0
$$

or

$$
x(0)=x^{\prime}(1)=0,
$$

where $\varphi_{p}(s)=|s|^{p-2} s, p>1$. When the nonlinear term $f$ is without dependence on the first order derivative, the existence and multiplicity results are available in the literature $[9,11,15,17,19]$. In [11], Kong and Wang studied the multiplicity for equation

$$
\left(\varphi_{p}\left(x^{\prime}(t)\right)\right)^{\prime}+q(t) f(t, x(t))=0,0<t<1,
$$

subject to some nonlinear boundary conditions by using the theory of fixed point index. In [17], Lü et al obtained triple positive solutions of Problem (1.4)-(1.2) and (1.4)-(1.3) by using the Leggett-Williams fixed point theorem. In [9, 15], He and Ge obtained triple positive solutions, Liu and Ge obtained twin positive solutions for Equation (1.4) with some nonlinear boundary conditions by using the Leggett-Williams fixed point theorem and a fixed point theorem due to Avery and Henderson, respectively. And in [19], by using the shooting method, Wong obtain the existence of positive solution for Equation (1.4) with boundary condition $x^{\prime}(0)=x(1)=0$. However, multiplicity are not available for the case that the nonlinear term is involved in first order derivative explicitly. This paper fills this gap in the literature. Equations of the above form occur in the study of the $n$-dimensional $p$-Laplace equation, non-Newtonian fluid theory and the turbulent flow of a gas in a porous medium [18]. We say $x(t)$ is a positive solution of above problem provided $x(t) \geq 0$ on $[0,1]$.

Throughout this paper, it is assumed that:

(C1) $f \in C([0,1] \times[0, \infty) \times R,[0, \infty))$;

(C2) $q(t)$ is nonnegative continuous function defined in $(0,1), q(t) \not \equiv 0$ on any subinterval of $(0,1)$. In addition, $0<\int_{0}^{1} q(t) d t<+\infty$.

Our main results will depend on an application of a fixed-point theorem due to Avery and Peterson which deals with fixed points of a cone-preserving operator defined on an ordered Banach space. The emphasis here is that the nonlinear term is involved explicitly with the first-order derivative.

\section{Background Materials and Definitions}

For the convenience of the reader, we present here the necessary definitions from cone theory in Banach spaces; these definitions can be found in the recent literature.

Definition 2.1. Let $E$ be a real Banach space over $R$. A nonempty convex closed set $P \subset E$ is said to be a cone provided that 
(i) $a u \in P$ for all $u \in P$ and all $a \geq 0$ and

(ii) $u,-u \in P$ implies $u=0$.

Every cone $P \subset E$ induces an ordering in $E$ given by

$$
x \leq y, \quad \text { if and only if } y-x \in P .
$$

Definition 2.2. An operator is called completely continuous, if it is continuous and maps bounded sets into precompact sets.

Definition 2.3. The map $\alpha$ is said to be a nonnegative continuous concave functional on a cone $P$ of a real Banach space $E$ provided that $\alpha: P \rightarrow[0, \infty)$ is continuous and

$$
\alpha(t x+(1-t) y) \geq t \alpha(x)+(1-t) \alpha(y)
$$

for all $x, y \in P$ and $0 \leq t \leq 1$. Similarly, we say the map $\gamma$ is a nonnegative continuous convex functional on a cone $P$ of a real Banach space $E$ provided that $\gamma: P \rightarrow[0, \infty)$ is continuous and

$$
\gamma(t x+(1-t) y) \leq t \gamma(x)+(1-t) \gamma(y)
$$

for all $x, y \in P$ and $0 \leq t \leq 1$.

Let $\gamma$ and $\theta$ be nonnegative continuous convex functionals on $P, \alpha$ be a nonnegative continuous concave functional on $P$, and $\psi$ be a nonnegative continuous functional on $P$. Then for positive real numbers $a, b, c$, and $d$, we define the following convex sets:

$$
\begin{aligned}
P(\gamma, d) & =\{x \in P \mid \gamma(x)<d\}, \\
P(\gamma, \alpha, b, d) & =\{x \in P \mid b \leq \alpha(x), \gamma(x) \leq d\}, \\
P(\gamma, \theta, \alpha, b, c, d) & =\{x \in P \mid b \leq \alpha(x), \theta(x) \leq c, \gamma(x) \leq d\},
\end{aligned}
$$

and a closed set

$$
R(\gamma, \psi, a, d)=\{x \in P \mid a \leq \psi(x), \gamma(x) \leq d\} .
$$

The following fixed-point theorem due to Avery and Peterson is fundamental in the proofs of our main results.

Theorem 2.1.([6]) Let $P$ be a cone in a real Banach space E. Let $\gamma$ and $\theta$ be nonnegative continuous convex functionals on $P, \alpha$ be a nonnegative continuous concave functional on $P$, and $\psi$ be a nonnegative continuous functional on $P$ satisfying $\psi(\lambda x) \leq$ $\lambda \psi(x)$ for $0 \leq \lambda \leq 1$, such that for some positive numbers $M$ and $d$,

$$
\alpha(x) \leq \psi(x) \quad \text { and } \quad\|x\| \leq M \gamma(x),
$$

for all $x \in \overline{P(\gamma, d)}$. Suppose

$$
T: \overline{P(\gamma, d)} \rightarrow \overline{P(\gamma, d)}
$$

is completely continuous and there exist positive numbers $a, b$, and $c$ with $a<b$ such that 
(S1) $\{x \in P(\gamma, \theta, \alpha, b, c, d) \mid \alpha(x)>b\} \neq \emptyset, \alpha(T x)>b$ for $x \in P(\gamma, \theta, \alpha, b, c, d)$;

(S2) $\alpha(T x)>b$ for $x \in P(\gamma, \alpha, b, d)$ with $\theta(T x)>c$;

(S3) $0 \notin R(\gamma, \psi, a, d)$ and $\psi(T x)<a$ for $x \in R(\gamma, \psi, a, d)$ with $\psi(x)=a$.

Then $T$ has at least three fixed points $x_{1}, x_{2}, x_{3} \in \overline{P(\gamma, d)}$, such that

$$
\begin{gathered}
\gamma\left(x_{i}\right) \leq d, \quad \text { for } i=1,2,3 ; \\
b<\alpha\left(x_{1}\right) ; \\
a<\psi\left(x_{2}\right), \quad \text { with } \alpha\left(x_{2}\right)<b ;
\end{gathered}
$$

and

$$
\psi\left(x_{3}\right)<a .
$$

\section{Existence of Triple Positive Solutions to Problem (1.1) - (1.2)}

In this section, we impose growth conditions on $f$ which allow us to apply Theorem 2.1 to establish the existence of triple positive solutions of Problem (1.1)-(1.2). It follows from (C2) that there exists a natural number $k \geq 3$ such that $0<\int_{1 / k}^{(k-1) / k} q(t) d t<+\infty$.

Let $X=C^{1}[0,1]$ be endowed with the ordering $x \leq y$ if $x(t) \leq y(t)$ for all $t \in[0,1]$, and the maximum norm, $\|x\|=\max \left\{\max _{0 \leq t \leq 1}|x(t)|, \max _{0 \leq t \leq 1}\left|x^{\prime}(t)\right|\right\}$. From the fact $\left(\varphi_{p}\left(x^{\prime}(t)\right)\right)^{\prime}=-q(t) f\left(t, x(t), x^{\prime}(t)\right) \leq 0$, we know that $x$ is concave on $[0,1]$. So, define the cone $P_{1} \subset X$ by

$$
P_{1}=\{x \in X \mid x(t) \geq 0, x(0)=x(1)=0, x \text { is concave on }[0,1]\} \subset X .
$$

Let the nonnegative continuous concave functional $\alpha_{1}$, the nonnegative continuous convex functional $\theta_{1}, \gamma_{1}$, and the nonnegative continuous functional $\psi_{1}$ be defined on the cone $P_{1}$ by

$\gamma_{1}(x)=\max _{0 \leq t \leq 1}\left|x^{\prime}(t)\right|, \quad \psi_{1}(x)=\theta_{1}(x)=\max _{0 \leq t \leq 1}|x(t)|, \quad \alpha_{1}(x)=\min _{\frac{1}{k} \leq t \leq \frac{k-1}{k}}|x(t)|, \quad$ for $x \in P_{1}$.

Lemma 3.1. If $x \in P_{1}$, then

$$
\max _{0 \leq t \leq 1}|x(t)| \leq \frac{1}{2} \max _{0 \leq t \leq 1}\left|x^{\prime}(t)\right| .
$$

Proof. Suppose by contrary that there exist $t_{0} \in(0,1)$ such that $\left|x\left(t_{0}\right)\right|>\frac{1}{2} \max _{0 \leq t \leq 1}$ $\left|x^{\prime}(t)\right|=: A$, then by the Roll Mid-Value Theorem there exist $t_{1} \in\left(0, t_{0}\right), t_{2} \in\left(t_{0}, 1\right)$ such that

$$
x^{\prime}\left(t_{1}\right)=\frac{x\left(t_{0}\right)-x(0)}{t_{0}}=\frac{x\left(t_{0}\right)}{t_{0}}, \quad x^{\prime}\left(t_{2}\right)=\frac{x(1)-x\left(t_{0}\right)}{1-t_{0}}=\frac{-x\left(t_{0}\right)}{1-t_{0}} .
$$


Thus, $\max _{0 \leq t \leq 1}\left|x^{\prime}(t)\right| \geq \max \left\{\left|x^{\prime}\left(t_{1}\right)\right|,\left|x^{\prime}\left(t_{2}\right)\right|\right\}>2 A$, a contradiction. The proof is complete.

With Lemma 3.1, the definitions of functionals, and the concavity of $x$, the functionals defined above hold relations

$$
\frac{1}{k} \theta_{1}(x) \leq \alpha_{1}(x) \leq \theta_{1}(x)=\psi_{1}(x), \quad\|x\|=\max \left\{\theta_{1}(x), \gamma_{1}(x)\right\}=\gamma_{1}(x),
$$

for all $x \in \overline{P_{1}\left(\gamma_{1}, d\right)} \subset P_{1}$, and the condition (2.1) is satisfied.

Define an operator $T: P_{1} \rightarrow P_{1}$ by

$$
(T x)(t):= \begin{cases}\int_{0}^{t} \varphi_{p}^{-1}\left(\int_{s}^{\sigma} q(r) f\left(r, x(r), x^{\prime}(r)\right) d r\right) d s, & \text { for } 0 \leq t \leq \sigma \\ \int_{t}^{1} \varphi_{p}^{-1}\left(\int_{\sigma}^{s} q(r) f\left(r, x(r), x^{\prime}(r)\right) d r\right) d s, & \text { for } \sigma \leq t \leq 1\end{cases}
$$

where $\sigma\left(=\sigma_{x}\right)$ is described as follows. Let

$$
\begin{aligned}
y(t):= & \int_{0}^{t} \varphi_{p}^{-1}\left(\int_{s}^{t} q(r) f\left(r, x(r), x^{\prime}(r)\right) d r\right) d s \\
& -\int_{t}^{1} \varphi_{p}^{-1}\left(\int_{t}^{s} q(r) f\left(r, x(r), x^{\prime}(r)\right) d r\right) d s, \quad 0<t<1 .
\end{aligned}
$$

Clearly, $y(t)$ is continuous and strictly increasing in $(0,1)$ and $y(0+)<0<y(1-)$. Thus $y(t)$ has zeros in $(0,1)$, and let $\sigma$ be a zero of $y(t)$ in $(0,1)$.

From the definition of $T$, we deduce that for each $x \in P_{1}, T x \in C^{1}[0,1]$, and satisfies (1.2). Moreover, $(T x)(\sigma)$ is the maximum value of $T x$ on $[0,1]$, since

$$
(T x)^{\prime}(t):= \begin{cases}\varphi_{p}^{-1}\left(\int_{t}^{\sigma} q(r) f\left(r, x(r), x^{\prime}(r)\right) d r\right), & \text { for } 0 \leq t \leq \sigma \\ -\varphi_{p}^{-1}\left(\int_{\sigma}^{t} q(r) f\left(r, x(r), x^{\prime}(r)\right) d r\right), & \text { for } \sigma \leq t \leq 1\end{cases}
$$

is continuous and nonincreasing in $[0,1]$ and $(T x)^{\prime}(\sigma)=0$. Since $(T x)^{\prime}$ is nonincreasing on $[0,1]$, we have $T x \in P_{1}$. Moreover,

$$
\left(\varphi_{p}\left((T x)^{\prime}(t)\right)\right)^{\prime}+q(t) f\left(t,(T x)(t),(T x)^{\prime}(t)\right)=0,0<t<1 .
$$

This show that $T\left(P_{1}\right) \subset P_{1}$, and that each fixed point of $T$ is a solution of Problem (1.1)-(1.2). By [17, 19], $T: P_{1} \rightarrow P_{1}$ is completely continuous.

Letting

$$
\begin{aligned}
& C_{1}=\varphi_{p}^{-1}\left(\int_{0}^{1} q(r) d r\right), \\
& M_{1}=\max \left\{\int_{0}^{\frac{1}{2}} \varphi_{p}^{-1}\left(\int_{s}^{\frac{1}{2}} q(r) d r\right) d s, \int_{\frac{1}{2}}^{1} \varphi_{p}^{-1}\left(\int_{\frac{1}{2}}^{s} q(r) d r\right) d s\right\}, \\
& N_{1}=\min \left\{\int_{\frac{1}{k}}^{\frac{1}{2}} \varphi_{p}^{-1}\left(\int_{s}^{\frac{1}{2}} q(r) d r\right) d s, \int_{\frac{1}{2}}^{\frac{k-1}{k}} \varphi_{p}^{-1}\left(\int_{\frac{1}{2}}^{s} q(r) d r\right) d s\right\} .
\end{aligned}
$$


Theorem 3.1. Suppose (C1), (C2) hold and there exist constants $0<a<b \leq d / 2 k$ such that the following assumptions hold

(A1) $f(t, u, v) \leq \varphi_{p}\left(\frac{d}{C_{1}}\right)$, for $(t, u, v) \in[0,1] \times[0, d / 2] \times[-d, d]$;

(A2) $f(t, u, v)>\varphi_{p}\left(\frac{k b}{N_{1}}\right)$, for $(t, u, v) \in[1 / k,(k-1) / k] \times[b, k b] \times[-d, d]$;

(A3) $f(t, u, v)<\varphi_{p}\left(\frac{a}{M_{1}}\right)$, for $(t, u, v) \in[0,1] \times[0, a] \times[-d, d]$.

Then, the boundary value problem (1.1)-(1.2) has at least three positive solutions $x_{1}, x_{2}$, and $x_{3}$ satisfying

$$
\begin{gathered}
\max _{0 \leq t \leq 1}\left|x_{i}^{\prime}(t)\right| \leq d, \quad \text { for } i=1,2,3 \\
b<\min _{\frac{1}{k} \leq t \leq \frac{k-1}{k}}\left|x_{1}(t)\right| \\
a<\max _{0 \leq t \leq 1}\left|x_{2}(t)\right|<k b, \quad \text { with } \min _{\frac{1}{k} \leq t \leq \frac{k-1}{k}}\left|x_{2}(t)\right|<b ;
\end{gathered}
$$

and

$$
\max _{0 \leq t \leq 1}\left|x_{3}(t)\right|<a
$$

Proof. Problem (1.1)-(1.2) has a solution $x=x(t)$ if and only if $x$ solves the operator equation $x(t)=T x(t)$. It is well know that $T: P_{1} \rightarrow P_{1}$ is completely continuous.

We now show that all the conditions of Theorem 2.1 are satisfied.

If $x \in \overline{P_{1}\left(\gamma_{1}, d\right)}$, then there is $\gamma_{1}(x)=\max _{0 \leq t \leq 1}\left|x^{\prime}(t)\right| \leq d$. With Lemma 3.1, $\max _{0 \leq t \leq 1}|x(t)| \leq \frac{d}{2}$, then assumption (A1) implies $f\left(t, x(t), x^{\prime}(t)\right) \leq \varphi_{p}\left(\frac{d}{C_{1}}\right)$. On the other hand, for $x \in P_{1}$, there is $T x \in P_{1}$, then $T x$ is concave on $[0,1]$, and $\max _{t \in[0,1]}\left|(T x)^{\prime}(t)\right|=\max \left\{\left|(T x)^{\prime}(0)\right|,\left|(T x)^{\prime}(1)\right|\right\}$, so

$$
\begin{aligned}
\gamma_{1}(T x) & =\max _{t \in[0,1]}\left|(T x)^{\prime}(t)\right| \\
& =\max \left\{\varphi_{p}^{-1}\left(\int_{0}^{\sigma} q(r) f\left(r, x(r), x^{\prime}(r)\right) d r\right), \varphi_{p}^{-1}\left(\int_{\sigma}^{1} q(r) f\left(r, x(r), x^{\prime}(r)\right) d r\right)\right\} \\
& \leq \frac{d}{C_{1}} \cdot \varphi_{p}^{-1}\left(\int_{0}^{1} q(r) d r\right) \\
& =\frac{d}{C_{1}} \cdot C_{1}=d .
\end{aligned}
$$

Hence, $T: \overline{P_{1}\left(\gamma_{1}, d\right)} \rightarrow \overline{P_{1}\left(\gamma_{1}, d\right)}$.

To check condition (S1) of Theorem 2.1, we choose $x(t)=k b / 2,0 \leq t \leq 1$. It is easy to see that $x(t)=k b / 2 \in P_{1}\left(\gamma_{1}, \theta_{1}, \alpha_{1}, b, k b, d\right)$ and $\alpha_{1}(x)=\alpha_{1}(k b / 2)>b$, and so $\left\{x \in P_{1}\left(\gamma_{1}, \theta_{1}, \alpha_{1}, b, k b, d\right) \mid \alpha_{1}(x)>b\right\} \neq \emptyset$. Hence, if $x \in P_{1}\left(\gamma_{1}, \theta_{1}, \alpha_{1}, b, k b, d\right)$, then $b \leq x(t) \leq k b,\left|x^{\prime}(t)\right| \leq d$ for $1 / k \leq t \leq(k-1) / k$. From assumption (A2), we have 
$f\left(t, x(t), x^{\prime}(t)\right) \geq \varphi_{p}\left(\frac{k b}{N_{1}}\right)$ for $1 / k \leq t \leq(k-1) / k$, and by the conditions of $\alpha_{1}$ and the cone $P_{1}$, we have

$$
\begin{aligned}
\alpha_{1}(T x)= & \min _{\frac{1}{k} \leq t \leq \frac{k-1}{k}}(T x)(t) \\
\geq & \frac{1}{k} \max _{0 \leq t \leq 1}|(T x)(t)| \\
= & \frac{1}{k} \int_{0}^{\sigma} \varphi_{p}^{-1}\left(\int_{s}^{\sigma} q(r) f\left(r, x(r), x^{\prime}(r)\right) d r\right) d s \\
= & \frac{1}{k} \int_{\sigma}^{1} \varphi_{p}^{-1}\left(\int_{\sigma}^{s} q(r) f\left(r, x(r), x^{\prime}(r)\right) d r\right) d s \\
\geq & \frac{1}{k} \cdot \min \left\{\int_{0}^{\frac{1}{2}} \varphi_{p}^{-1}\left(\int_{s}^{\frac{1}{2}} q(r) f\left(r, x(r), x^{\prime}(r)\right) d r\right) d s\right. \\
& \left.\int_{\frac{1}{2}}^{1} \varphi_{p}^{-1}\left(\int_{\frac{1}{2}}^{s} q(r) f\left(r, x(r), x^{\prime}(r)\right) d r\right) d s\right\} \\
\geq & \frac{1}{k} \cdot \min \left\{\int_{\frac{1}{k}}^{\frac{1}{2}} \varphi_{p}^{-1}\left(\int_{s}^{\frac{1}{2}} q(r) f\left(r, x(r), x^{\prime}(r)\right) d r\right) d s\right. \\
& \left.\int_{\frac{1}{2}}^{\frac{k-1}{k}} \varphi_{p}^{-1}\left(\int_{\frac{1}{2}}^{s} q(r) f\left(r, x(r), x^{\prime}(r)\right) d r\right) d s\right\} \\
\geq & \frac{1}{k} \cdot \frac{k b}{N_{1}} \cdot N_{1}=b .
\end{aligned}
$$

i.e.,

$$
\alpha_{1}(T x)>b, \text { for all } x \in P_{1}\left(\gamma_{1}, \theta_{1}, \alpha_{1}, b, k b, d\right) \text {. }
$$

This show that condition (S1) of Theorem 2.1 is satisfied.

Secondly, with (3.1) and $b \leq \frac{d}{2 k}$, we have

$$
\alpha_{1}(T x) \geq \frac{1}{k} \theta_{1}(T x)>\frac{1}{k} \cdot k b=b, \text { for all } x \in P_{1}\left(\gamma_{1}, \alpha_{1}, b, d\right) \text { with } \theta_{1}(T x)>k b
$$

Thus, condition (S2) of Theorem 2.1 is satisfied.

We finally show that (S3) of Theorem 2.1 also holds. Clearly, as $\psi_{1}(0)=0<a$, there holds that $0 \notin R\left(\gamma_{1}, \psi_{1}, a, d\right)$. Suppose that $x \in R\left(\gamma_{1}, \psi_{1}, a, d\right)$ with $\psi_{1}(x)=a$. Then, 
by the assumption (A3),

$$
\begin{aligned}
\psi_{1}(T x)= & \max _{0 \leq t \leq 1}|(T x)(t)| \\
= & \int_{0}^{\sigma} \varphi_{p}^{-1}\left(\int_{s}^{\sigma} q(r) f\left(r, x(r), x^{\prime}(r)\right) d r\right) d s \\
= & \int_{\sigma}^{1} \varphi_{p}^{-1}\left(\int_{\sigma}^{s} q(r) f\left(r, x(r), x^{\prime}(r)\right) d r\right) d s \\
\leq & \max \left\{\int_{0}^{\frac{1}{2}} \varphi_{p}^{-1}\left(\int_{s}^{\frac{1}{2}} q(r) f\left(r, x(r), x^{\prime}(r)\right) d r\right) d s,\right. \\
& \left.\int_{\frac{1}{2}}^{1} \varphi_{p}^{-1}\left(\int_{\frac{1}{2}}^{s} q(r) f\left(r, x(r), x^{\prime}(r)\right) d r\right) d s\right\} \\
< & \frac{a}{M_{1}} \cdot \max \left\{\int_{0}^{\frac{1}{2}} \varphi_{p}^{-1}\left(\int_{s}^{\frac{1}{2}} q(r) d r\right) d s, \int_{\frac{1}{2}}^{1} \varphi_{p}^{-1}\left(\int_{\frac{1}{2}}^{s} q(r) d r\right) d s\right\} \\
= & a .
\end{aligned}
$$

So, the condition (S3) of Theorem 2.1 is satisfied. On the other hand, for $x \in P_{1}$ there is (3.1) holds. Therefore, an application of Theorem 2.1 imply the boundary value problem (1.1)-(1.2) has at least three positive solutions $x_{1}, x_{2}$, and $x_{3}$ satisfying

$$
\begin{gathered}
\max _{0 \leq t \leq 1}\left|x_{i}^{\prime}(t)\right| \leq d, \quad \text { for } i=1,2,3 \\
b<\min _{\frac{1}{k} \leq t \leq \frac{k-1}{k}}\left|x_{1}(t)\right| \\
a<\max _{0 \leq t \leq 1}\left|x_{2}(t)\right|<k b, \quad \text { with } \min _{\frac{1}{k} \leq t \leq \frac{k-1}{k}}\left|x_{2}(t)\right|<b ;
\end{gathered}
$$

and

$$
\max _{0 \leq t \leq 1}\left|x_{3}(t)\right|<a
$$

The proof is complete.

Example 3.1. Consider the boundary value problem

$$
\begin{gathered}
\left(\left|x^{\prime}\right| x^{\prime}\right)^{\prime}+f\left(t, x(t), x^{\prime}(t)\right)=0, \quad 0<t<1, \\
x(0)=x(1)=0
\end{gathered}
$$

where

$$
f(t, u, v)= \begin{cases}\sin t+2306 \times u^{10}+\frac{1}{6}\left(\frac{v}{31000}\right)^{3} & \text { for } u \leq 4 \\ \sin t+2306 \times 4^{10}+\frac{1}{6}\left(\frac{v}{31000}\right)^{3} & \text { for } u>4\end{cases}
$$


Choose $a=\frac{1}{2}, b=1, k=4, d=50000$, we note $C_{1}=1, M_{1}=\sqrt{2} / 6, N_{1}=1 / 12$. Consequently, $f(t, u, v)$ satisfy

$$
\begin{aligned}
& f(t, u, v)<\varphi_{p}\left(\frac{a}{M_{1}}\right)=4.5, \text { for } 0 \leq t \leq 1,0 \leq u \leq \frac{1}{2},-50000 \leq v \leq 50000 \\
& f(t, u, v)>\varphi_{p}\left(\frac{4 b}{N_{1}}\right)=2304, \text { for } 1 / 4 \leq t \leq 3 / 4,1 \leq u \leq 4,-50000 \leq v \leq 50000 \\
& f(t, u, v)<\varphi_{p}\left(\frac{d}{C_{1}}\right)=2.5 \times 10^{9}, \text { for } 0 \leq t \leq 1,0 \leq u \leq 25000,-50000 \leq v \leq 50000 .
\end{aligned}
$$

Then all assumptions of Theorem 3.1 hold. Thus, with Theorem 3.1, Problem (3.3)-(3.4) has at least three positive solutions $x_{1}, x_{2}, x_{3}$ such that

$$
\begin{aligned}
\max _{0 \leq t \leq 1}\left|x_{i}^{\prime}(t)\right| & \leq 50000, \quad \text { for } i=1,2,3 \\
1 & <\min _{\frac{1}{4} \leq t \leq \frac{3}{4}}\left|x_{1}(t)\right| \\
\frac{1}{2}<\max _{0 \leq t \leq 1}\left|x_{2}(t)\right| & <4, \quad \text { with } \min _{\frac{1}{4} \leq t \leq \frac{3}{4}}\left|x_{2}(t)\right|<1 ;
\end{aligned}
$$

and

$$
\max _{0 \leq t \leq 1}\left|x_{3}(t)\right|<\frac{1}{2}
$$

Remark 3.1. The early results, see $[1,2,3,5,6,9,13]$, for example, are not applicable to the above problem. In conclusion, we see that the nonlinear term is involved in first derivative explicitly.

\section{Existence of Triple Positive Solutions to Problem (1.1) - (1.3)}

Now we deal with Problem (1.1)-(1.3). The method is just similar to what we have done above. Moreover, the solutions of Problem (1.1)-(1.3) are monotone increasing, which leads to the situation more simple, and so we leave the details to reader. Define the cone $P_{2} \subset X$ by

$$
P_{2}=\left\{x \in X \mid x(t) \geq 0, x(0)=x^{\prime}(1)=0, x \text { is concave on }[0,1]\right\} .
$$

Let the nonnegative continuous concave functional $\alpha_{2}$, the nonnegative continuous convex functional $\theta_{2}, \gamma_{2}$, and the nonnegative continuous functional $\psi_{2}$ be defined on the cone $P_{2}$ by

$$
\begin{gathered}
\gamma_{2}(x)=\max _{t \in[0,1]}\left|x^{\prime}(t)\right|=x^{\prime}(0), \quad \psi_{2}(x)=\theta_{2}(x)=\max _{t \in[0,1]}|x(t)|=x(1), \\
\alpha_{2}(x)=\min _{t \in\left[\frac{1}{k}, 1\right]}|x(t)|=x\left(\frac{1}{k}\right), \quad \text { for } x \in P_{2} .
\end{gathered}
$$


Lemma 4.1. If $x \in P_{2}$, then

$$
x(1) \leq x^{\prime}(0)
$$

With Lemma 4.1, the definition of functionals, and the concavity of $x$, the functionals defined above hold relations

$$
\frac{1}{k} \theta_{2}(x) \leq \alpha_{2}(x) \leq \theta_{2}(x)=\psi_{2}(x), \quad\|x\|=\max \left\{\theta_{2}(x), \gamma_{2}(x)\right\} \leq \gamma_{2}(x),
$$

for all $x \in \overline{P_{2}\left(\gamma_{2}, d\right)} \subset P_{2}$.

Define operator $T: P_{2} \rightarrow P_{2}$ by

$$
(T x)(t):=\int_{0}^{t} \varphi_{p}^{-1}\left(\int_{s}^{1} q(r) f\left(r, x(r), x^{\prime}(r)\right) d r\right) d s, \quad 0 \leq t \leq 1 .
$$

From the definition of $T$, there is for each $x \in P_{2}, T x \in P_{2}$ and $(T x)(1)$ is the maximum value of $T x$ on $[0,1]$. By $[17,19], T: P_{2} \rightarrow P_{2}$ is completely continuous.

Letting

$$
\begin{aligned}
C_{2} & =\varphi_{p}^{-1}\left(\int_{0}^{1} q(r) d r\right), \\
M_{2} & =\int_{0}^{1} \varphi_{p}^{-1}\left(\int_{s}^{1} q(r) d r\right) d s \\
N_{2} & =\int_{\frac{1}{k}}^{1} \varphi_{p}^{-1}\left(\int_{s}^{1} q(r) d r\right) d s .
\end{aligned}
$$

Theorem 4.1. Suppose (C1), (C2) hold and there exist constants $0<a<b \leq d / k$ such that the following assumptions hold

(B1) $f(t, u, v) \leq \varphi_{p}\left(\frac{d}{C_{2}}\right)$, for $(t, u, v) \in[0,1] \times[0, d] \times[-d, d]$;

(B2) $f(t, u, v)>\varphi_{p}\left(\frac{k b}{N_{2}}\right)$, for $(t, u, v) \in\left[\frac{1}{k}, 1\right] \times[b, k b] \times[-d, d]$;

(B3) $f(t, u, v)<\varphi_{p}\left(\frac{a}{M_{2}}\right)$, for $(t, u, v) \in[0,1] \times[0, a] \times[-d, d]$.

Then, the boundary value problem (1.1)-(1.3) has at least three positive solutions $x_{1}, x_{2}$, and $x_{3}$ satisfying

$$
\begin{aligned}
\max _{0 \leq t \leq 1}\left|x_{i}^{\prime}(t)\right| & \leq d, \quad \text { for } i=1,2,3 \\
b & <\min _{\frac{1}{k} \leq t \leq 1}\left|x_{1}(t)\right| \\
a<\max _{0 \leq t \leq 1}\left|x_{2}(t)\right| & <k b, \text { with } \min _{\frac{1}{k} \leq t \leq 1}\left|x_{2}(t)\right|<b ;
\end{aligned}
$$

and

$$
\max _{0 \leq t \leq 1}\left|x_{3}(t)\right|<a .
$$




\section{References}

[1] R. P. Agarwal, D. O'Regan and P. J. Y. Wong, Positive Solutions of Differential, Difference, and Integral Equations, Kluwer Academic Publishers, Boston, 1999.

[2] R. I. Avery, A generalization of the Leggett-Williams fixed point theorem, Math. Sci. Res. Hot-line 2 (1998), 9-14.

[3] R. I. Avery and D. R. Anderson, Fixed point theorem of cone expansion and compression of functional type, J. Diff. Equa. Appl. 8 (2002), 1073-1083.

[4] R. I. Avery and J. Henderson, Three sysmmetric positive solutions for a second-order boundary value problem, Appl. Math. Lett. 13 (2000), 1-7.

[5] R. I. Avery and J. Henderson, Two positive fixed points of nonlinear operators on ordered Banach spaces, (preprint).

[6] R. I. Avery and A. C. Peterson, Three positive fixed points of nonlinear operators on ordered Banach spaces, Comput. Math. Appl. 42 (2001), 313-322.

[7] Z. B. Bai and H. Y. Wang, On positive solutions of some nonlinear fourth-order beam equations, J. Math. Anal. Appl. 270 (2002), 357-368.

[8] D. Guo and V. Lakshmikantham, Nonlinear Problems in Abstract Cones, Academic Press, New York, 1988.

[9] X. M. He and W. G. Ge, Multiple positive solutions for one-dimensional p-Laplacian boundary value problems, Appl. Math. Lett. 15 (2002), 937-943.

[10] J. Henderson and H. Y. Wang, Positive solutions for nonlinear eigenvalue problems, J. Math. Anal. Appl. 208 (1997), 252-259.

[11] L. B. Kong and J. Y. Wang, Multiple positive solutions for the one-dimensional p-laplacian, Nonl. Anal. 42(2000), 1327-1333.

[12] M. A. Krasnosel'skii, Positive Solutions of Operator Equations, Noordhoff, Gronignen, 1964.

[13] R. W. Leggett and L. R. Williams, Multiple positive fixed points of nonlinear operators on ordered Banach spaces, Indiana Univ. Math. J. 28 (1979), 673-688.

[14] W. C. Lian, F. H. Wong, and C. C. Yeh, On the existence of positive solutions of nonlinear second order differential equations, Proc. Amer. Math. Soc. 124 (1996), 1117-1126.

[15] Y. J. Liu and W. G. Ge, Multiple positive solutions to a three-point boundary value problem with p-laplacian, J. Math. Anal. Appl. 277(2003), 293-302.

[16] Z. L. Liu and F. Y. Li, Multiple positive solutions of nonlinear two point boundary value problem, J. Math. Anal. Appl. 203 (1996), 610-625.

[17] H. S. Lü, D. O'Regan and C. K. Zhong, Multiple positive solutions for the one-dimensional singular p-laplacian, Appl. Math. Comp. 133 (2002), 407-422.

[18] D. O'Regan, Some general existence principles and results for $\left(\varphi\left(y^{\prime}\right)\right)^{\prime}=q f\left(t, y, y^{\prime}\right), 0<$ $t<1$, SIAM J. Math. Appl. 24 (1993), 648-668.

[19] F. H. Wong, Existence of positive solutions for m-laplacian boundary value problems, Appl. Math. Lett. 12 (1999), 11-17.

[20] E. Zeidler, Nonlinear Analysis and Its Applications I: Fixed-Point Theorems, SpringerVerlag, New York, 1993.

College of Information Science \& Technology, Shandong University of Science and Technology, Qingdao 266510, People's Republic of China.

E-mail: zhanbingbai@163.com

Department of Applied Mathematics, University of Petroleum, Dongying 257061, People's Republic of China.

College of Information Science \& Technology, Shandong University of Science and Technology, Qingdao 266510, People's Republic of China. 